\title{
Extradural Dermoid Cyst of the Parasellar Region: A Case Report
}

\author{
Hidenori Endo, M.D.,, ${ }^{1}$ Kensuke Murakami, M.D., ${ }^{1}$ Mika Watanabe, M.D., ${ }^{2}$ \\ and Teiji Tominaga, M.D. 1
}

Dermoid cysts are rare congenital intracranial tumors. Among these tumors, extradural localization is extremely rare. We report a patient with an extradural dermoid cyst of the right parasellar region, causing right visual disturbance. Computed tomography revealed a hypodense mass lesion with rimlike calcification at the right parasellar region, accompanying marked erosion of the adjacent skull base. The tumor appeared as a heterogeneous intensity on magnetic resonance imaging and was surrounded by thin gadolinium enhancement without inner enhancement. The right optic nerve was compressed by the tumor. Surgical resection was successfully performed using a right frontotemporal extradural approach. The entire tumor was completely resided extradurally and was enclosed by saclike, stretched dura mater and extended deeply into the skull base. Histopathologic findings were consistent with the features of dermoid cyst. The postoperative course was uneventful, and the visual disturbance improved. Neuroradiological features, strategies for surgical treatment, and mechanisms responsible for preoperative symptoms are discussed.

KEYWORDS: Intracranial dermoid cyst, extradural localization, parasellar region, surgical treatment

Intracranial dermoid cysts are rare tumors constituting less than $1 \%$ of all intracranial tumors. ${ }^{1}$ The tumors typically arise in infants to young adults because of their congenital origin ${ }^{2-4}$ and are relatively rare in middle-aged or older people. ${ }^{5}$ They are considered to be the product of a defective closure of the neural tube, which occurs between the third and fifth gestational week. ${ }^{4}$ Associated dermal sinuses cause earlier onset of clinical symptoms such as infection. ${ }^{4}$ Other common symptoms including headaches, seizures, and chemical meningitis, and visual disturbances occur late in the clinical course because of its slowgrowing nature. ${ }^{2,3,6}$ Many reports have mentioned the intradural posterior fossa and the midline as the preferential localization of these tumors. ${ }^{1,7}$ In contrast, extradural dermoid cysts are a much rarer entity., Here we report a case with an extradural dermoid cyst of the parasellar region, which was successfully treated with surgical resection.

\section{CASE REPORT}

A 54-year-old woman was admitted to our hospital with complaints of a 2-year history of right visual disturbance and chronic headache. Ophthalmologic evaluation revealed mild impairment of right visual acuity and

\footnotetext{
${ }^{1}$ Departments of Neurosurgery and ${ }^{2}$ Pathology, Tohoku University Graduate School of Medicine, Sendai, Miyagi, Japan.

Address for correspondence and reprint requests: Hidenori Endo, M.D., Department of Neurosurgery, Tohoku University Graduate School of Medicine, 1-1 Seiryo-machi, Aoba-ku, Sendai 980-8574, Japan (e-mail: hideendo@gmail.com).

Skull Base Rep 2011;1:3-6. Copyright (C) 2011 by Thieme Medical
}

Publishers, Inc., 333 Seventh Avenue, New York, NY 10001, USA. Tel: +1 (212) 584-4662.

Received: October 8, 2009. Accepted after revision: June 25, 2010. Published online: August 6, 2010.

DOI: http://dx.doi.org/10.1055/s-0030-1263284.

ISSN 2157-6971. 

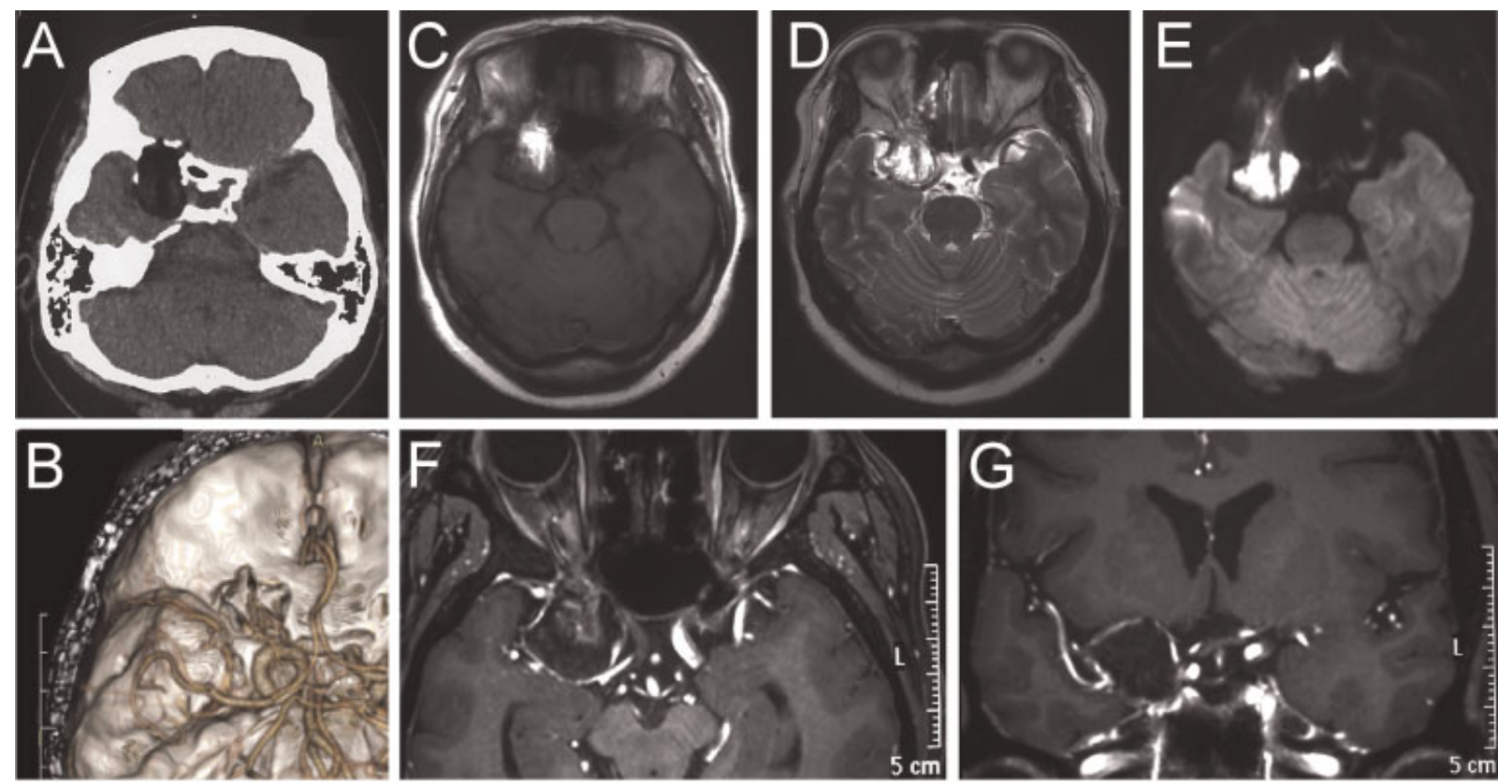

Figure 1 Radiological findings on initial presentation. Computed tomography (CT) showing the tumor as a hypodensity with rimlike calcification at the right parasellar region (A). Three-dimensional CT angiography demonstrating the skull base erosion and the compressed intracranial major vessels (B). Axial T1- (C) and T2-weighted magnetic resonance imaging (MRI) (D) demonstrating a heterogeneous mass lesion. Diffusion-weighted image showing the tumor as marked hyperintensity (E). Axial (F) and coronal (G) fat-suppression T1-weighted MRI with gadolinium enhancement revealing thin enhancement of the tumor rim, which continued to the dura mater.

abnormality of right color vision. Visual field examination revealed abnormal enlargement of paracentral scotoma, relative scotoma, and blind spot of Mariotte. Fundus examination showed right pale optic papilla. Eye movement was normal.

Computed tomography $(\mathrm{CT})$ revealed a hypodense mass lesion with rimlike calcification at the right parasellar region (Fig. 1A). The lesion eroded the medial aspect of the right sphenoidal ridge, the right anterior clinoid process, and the lateral wall of the right optic canal. Three-dimensional CT angiography showed posteriorly compressed internal carotid artery, M1 segment of the right middle cerebral artery (MCA), and $\mathrm{A} 1$ segment of the right anterior cerebral artery (Fig. 1B). The tumor was shown as a heterogeneous mass on conventional magnetic resonance imaging (MRI) with marked hyperintensity on diffusionweighted imaging (Fig. 1C, 1D, 1E). The tumor was surrounded by thin gadolinium enhancement without inner enhancement (Fig. 1F and $1 \mathrm{G}$ ). The posteriorly compressed major vessels, such as MCA, were not encased in the tumor. The right optic nerve was compressed by the tumor.

After insertion of a lumbar drain to minimize intraoperative brain retraction, right frontotemporal craniotomy was performed to widely expose both the anterior and middle cranial fossa. The yellowish, soft, and waxy tumor was exposed extradurally, which directly eroded the right anterior clinoid process and medial sphenoidal ridge and extended into the ethmoid sinus (Fig. 2A). The entire tumor resided extradurally and was enclosed by the saclike, stretched dura mater. The thin cyst wall adhering to the outer dura was removed as much as possible. As the tumor was removed inward piece by piece, the right optic nerve was imperceptibly revealed via eroded optic canal and optic nerve sheath (Fig. 2B). The eroded right optic nerve sheath was entirely surrounded by the tumor. Following the resection of the extradural part, the inside of the dura was inspected. The M1 portion of the right MCA and the basal aspect of the right frontal lobe were abnormally and tightly adhered to the upward projecting inner dura mater (Fig. 2C). However, no intradural mass lesion was observed (Fig. 2D).

Histopathologic examination of the surgical specimen showed fragments of keratinized tissue with hair follicles, adipose tissue, sebaceous glands, and sweat glands, which was consistent with the histopathologic features of dermoid cyst. The postoperative course was uneventful, and the patient was discharged 12 days after surgery. Ophthalmologic examination at discharge revealed improvement of visual field defect and abnormal color vision compared with the preoperative assessment, though right visual acuity was unchanged. MRI performed 3 months after discharge confirmed resection of the tumor without any surgical complications (Fig. 3). 

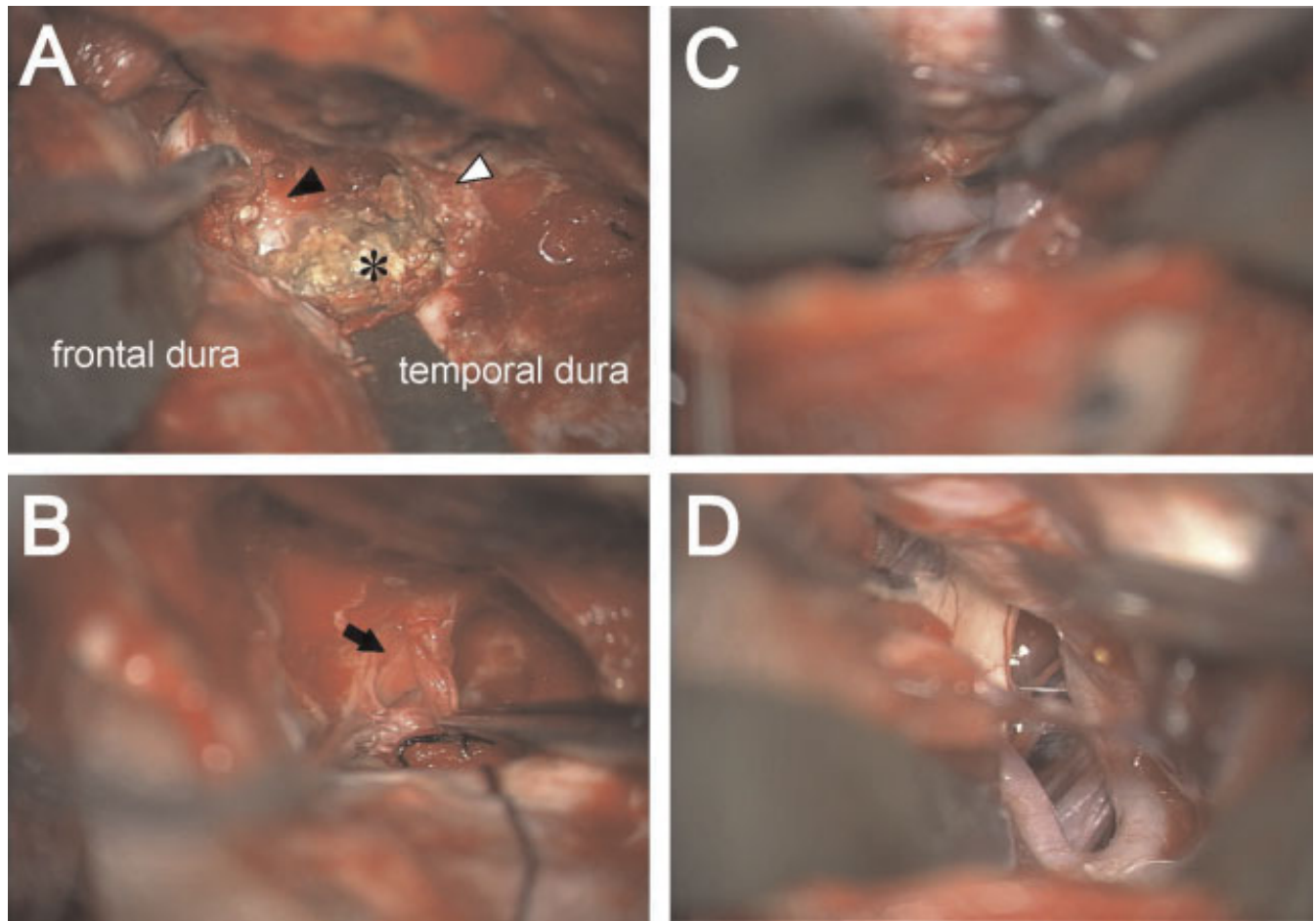

Figure 2 Intraoperative view. (A) The extradural tumor (black asterisk) enclosed by saclike, stretched dura. Black arrowhead and white arrowhead showing the lateral wall of the right cavernous sinus and meningo-orbital band, respectively. (B) Right optic nerve (black arrow) revealed via eroded optic canal and optic nerve sheath. (C) Intradural inspection. Firm adhesion between right M1 and inner surface of thickened dura mater. (D) No intradural tumor observed through right sylvian fissure.
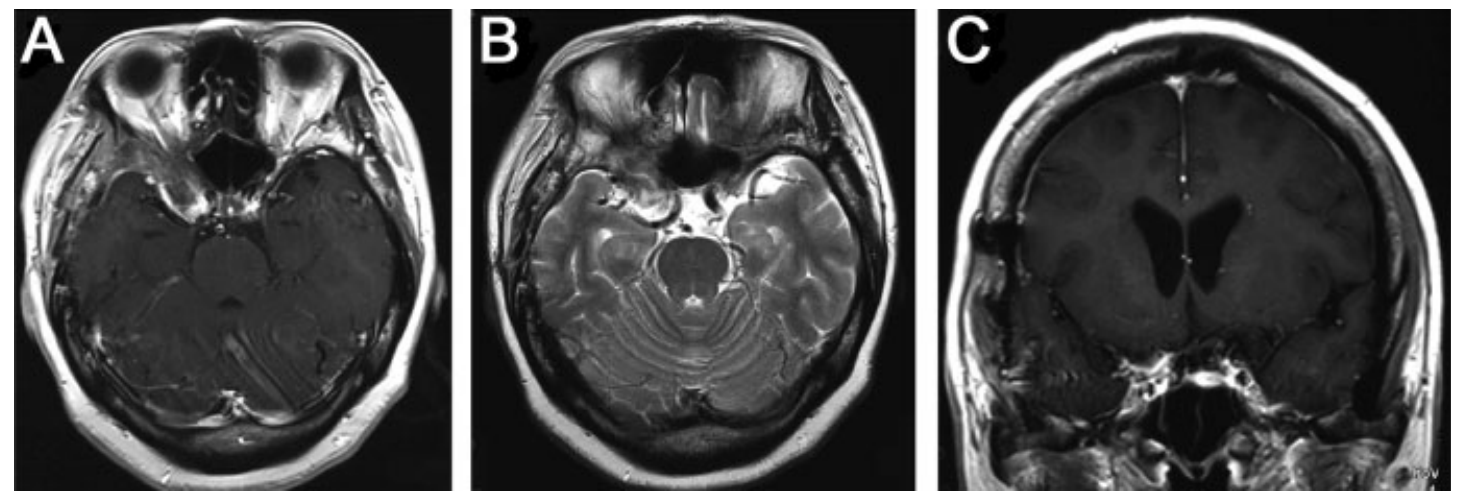

Figure 3 Postoperative axial T1-weighted magnetic resonance imaging (MRI) with gadolinium enhancement (A), T2weighted $\mathrm{MRI}(\mathrm{B})$, and coronal T1-weighted MRI with gadolinium enhancement $(\mathrm{C}) 3$ months after surgery showing no residual tumor.

\section{DISCUSSION}

The present case is quite unique because the tumor was completely extradural at the parasellar region. A noteworthy feature of preoperative CT was skull base erosion. A few cases with extradural skull base dermoid cysts reported in the past had a tendency to accompany skull base erosion. ${ }^{5,8}$ In contrast, skull base erosion is not involved in the intradural tumors. ${ }^{3}$ Another diagnostic key for the extradural localization is the MRI finding. The thin gadolinium enhancement surrounded the heterogeneous-intense tumor in the initial MRI. This enhancement was observed to continue to dura mater, suggesting the extradural localization of the tumor. Recent publications have indicated supratentorial lateral localization of the dermoid cysts such as intradural intraparenchymal position, ${ }^{3,9}$ extradural frontobasal region, ${ }^{9}$ extradural petrous apex region, ${ }^{5}$ and interdural region of the cavernous sinus. ${ }^{2,3}$ Most of these tumors, including the present case, arose from the adjacent area and seemed to have a similar developmental background. ${ }^{3,4}$ However, spatial relationships between the tumors and the dura mater are slightly different for each 
case. Identification of detailed tumor localization should be informative to determine operative approach. Neuroradiological findings of the present case can be preoperative diagnostic clues for extradural localization.

Dermoid cysts are surgically curable tumors like other benign brain tumors with low growth potential. ${ }^{2-4}$ The extradural approach was an appropriate choice for the present case, considering the extradural localization. Intraoperative findings showed that the tumor directly and deeply eroded the skull base. The tumor itself was totally removed. Adjacent skull base structures such as anterior clinoid process, optic strut, and optic canal roof were partially removed by drilling for sufficient optic nerve decompression, without complications such as cranial nerve palsy and cerebrospinal fluid rhinorrhea. These structures should have been more aggressively removed to aim for complete resection using an imageguided neuronavigation system, which could be a useful tool to identify anatomic relationships and remove the skull base tumor safely. Though the resection should be as radical as possible to avoid tumor recurrence, total resection is sometimes difficult because dermoid cysts tend to adhere tightly to the surrounding structures. ${ }^{2,4}$ In the present case, subtotal resection to avoid injuries of surrounding vital structures was justified, considering the low risk for tumor recurrence., ${ }^{2,3}$

Visual field defect and abnormal color vision of the right eye improved after surgery, though right visual acuity did not change. One of the possible explanations for this partial recovery after sufficient mass reduction might be the involvement of inflammation in the optic neuropathy. Ulrich observed a reaction of the surrounding structures to the epidermoid cysts with "hyalinization and thickening of the leptomeningeal connective tissue, infiltration of the meninges with foreign-body giant cells, histiocytes and lymphocytes, and fibrillary gliosis." ${ }^{10}$ In the present case, the right MCA tightly adhered to the inner dura of the middle cranial fossa. At the right frontal base, part of the extradural tumor penetrated both dura and pia mater at the point of adhesion. These findings might indicate that the inflammatory reaction caused by the extradural dermoid cyst could spread into the optic nerve beyond the dural structure such as the optic nerve sheath, resulting in refractory visual disturbance, though it was not histologically proven.

\section{CONCLUSION}

We described a rare case of extradural dermoid cyst of the parasellar region. Multimodal neuroradiological examination can be useful to identify extradural localization, which is necessary to determine a surgical approach. Safe surgical resection without injuries of surrounding vital structures is a goal of surgery, considering the low risk for recurrence. Visual disturbance, partially recovered after sufficient mass reduction, might be associated with optic nerve compression as well as inflammatory reaction caused by the dermoid cyst.

\section{REFERENCES}

1. Guidetti B, Gagliardi FM. Epidermoid and dermoid cysts. Clinical evaluation and late surgical results. J Neurosurg 1977; 47:12-18

2. Akdemir G, Dağlioğlu E, Ergüngör MF. Dermoid lesion of the cavernous sinus: case report and review of the literature. Neurosurg Rev 2004;27:294-298

3. Lunardi P, Missori P. Supratentorial dermoid cysts. J Neurosurg 1991;75:262-266

4. Caldarelli M, Massimi L, Kondageski C, Di Rocco C. Intracranial midline dermoid and epidermoid cysts in children. J Neurosurg 2004;100(5 Suppl Pediatrics):473-480

5. Ammirati M, Delgado M, Slone HW, Ray-Chaudhury A. Extradural dermoid tumor of the petrous apex. Case report. J Neurosurg 2007;107:426-429

6. Rubin G, Scienza R, Pasqualin A, Rosta L, Da Pian R. Craniocerebral epidermoids and dermoids. A review of 44 cases. Acta Neurochir (Wien) 1989;97:1-16

7. Bogdanowicz WM, Wilson DH. Dermoid cyst of the fourth ventricle demonstrated on brain scan. Case report. J Neurosurg 1972;36:228-230

8. Blythe JN, Revington PJ, Nelson R. Anterior cranial fossa dermoid cyst: case report. Br J Oral Maxillofac Surg 2007; 45:661-663

9. Civit T, Pinelli C, Lescure JP, Anxionnat R, Auque J, Hepner H. Stroke related to a dermoid cyst: case report. Neurosurgery 1997;41:1396-1399

10. Ulrich J. Intracranial epidermoids. A study on their distribution and spread. J Neurosurg 1964;21:1051-1058 